\title{
Research Article \\ Existence of Positive Solutions for a Class of Delay Fractional Differential Equations with Generalization to $\mathbf{N}$-Term
}

\author{
Azizollah Babakhani ${ }^{1}$ and Dumitru Baleanu' ${ }^{2,3}$ \\ ${ }^{1}$ Faculty of Basic Science, Babol University of Technology, Babol 47148-71167, Iran \\ ${ }^{2}$ Department of Mathematics and Computer Science, Faculty of Arts and Sciences Cankaya University, \\ 06530 Balgat, Ankara, Turkey \\ ${ }^{3}$ Institute of Space Sciences, P.O. Box, MG-23, RO 76900 Magurele-Bucharest, Romania
}

Correspondence should be addressed to Azizollah Babakhani, babakhani@nit.ac.ir

Received 25 July 2010; Accepted 17 March 2011

Academic Editor: Yoshikazu Giga

Copyright (C 2011 A. Babakhani and D. Baleanu. This is an open access article distributed under the Creative Commons Attribution License, which permits unrestricted use, distribution, and reproduction in any medium, provided the original work is properly cited.

We established the existence of a positive solution of nonlinear fractional differential equations $\mathfrak{L}(D)[x(t)-x(0)]=f\left(t, x_{t}\right), t \in(0, b]$ with finite delay $x(t)=\omega(t), t \in[-\tau, 0]$, where $\lim _{t \rightarrow 0} f\left(t, x_{t}\right)=$ $+\infty$, that is, $f$ is singular at $t=0$ and $x_{t} \in C\left([-\tau, 0], \mathbb{R}^{\geq 0}\right)$. The operator of $\mathfrak{L}(D)$ involves the Riemann-Liouville fractional derivatives. In this problem, the initial conditions with fractional order and some relations among them were considered. The analysis rely on the alternative of the Leray-Schauder fixed point theorem, the Banach fixed point theorem, and the Arzela-Ascoli theorem in a cone.

\section{Introduction}

Fractional differential equations have gained considerable importance due to their varied applications in viscoelasticity, electroanalytical chemistry, and many other physical problems [1-7]. So far there have been several fundamental works done on the fractional derivative and fractional differential equations $[1-4,6]$. These works are in the introduction of the theory of the fractional derivative and fractional differential equations and provide a systematic understanding of the fractional calculus such as the existence and the uniqueness of some analytic methods for solving fractional differential equations, namely, the Green's function method, the Mellin transform method, and the power series. 
The existence of positive solutions for fractional differential equations with delay has been carried out by various researchers [8-17]. In [8] the authors have investigated the following type of fractional differential equations:

$$
\begin{gathered}
\mathcal{L}(D)[x(t)-x(0)]=f\left(t, x_{t}\right), \quad t \in(0, T], \\
x(t)=\phi(t) \geq 0, \quad t \in[-w, 0],
\end{gathered}
$$

where

$$
\begin{gathered}
\mathcal{L}(D)=D^{\alpha_{n}}-\sum_{j=1}^{n-1} p_{j}(t) D^{\alpha_{n-j}}, \quad 0<\alpha_{1}<\cdots<\alpha_{n}<1, p_{j}(t)=\sum_{k=0}^{N_{j}} a_{j k} t^{k}, \\
p_{j}^{(2 m)}(t) \geq 0, \quad p_{j}^{(2 m+1)}(t) \leq 0, \quad m=0,1, \ldots,\left[\frac{N_{j}}{2}\right], j=1,2, \ldots, n-1,
\end{gathered}
$$

and $D^{\alpha_{j}}$ is the standard Riemann-Liouville fractional derivative, $T>0, w>0, \phi \in C=$ $C\left([-w, 0], \mathbb{R}^{+}\right)$and $f: I \times C \rightarrow \mathbb{R}^{+}$is a given continuous function, $I=[0, T]$.

As a pursuit of this in this paper, we discuss the existence of positive solutions for initial nonlinear fractional differential equations with finite delay,

$$
\begin{gathered}
\mathcal{L}(D)[x(t)-x(0)]=f\left(t, x_{t}\right), \quad t \in(0, b], \\
x(t)=\omega(t), \quad t \in[-\tau, 0],
\end{gathered}
$$

with initial conditions $x(0)=x_{0},\left[D^{\alpha-n+1} x(t)\right]_{t=0}=x_{n-1}$, and $\left[D^{\alpha-n+j} x(t)\right]_{t=0}=x_{n-j}$, where $\mathcal{L}(D)=D^{\alpha}-\sum_{j=1}^{n-1} a_{j} D^{\alpha-j}, a_{j}>0$, for all $j, j=1,2, \ldots, n-1, n-1<\alpha \leq n, n \in \mathbb{N}, f$ : $(0, b] \times C \rightarrow[0,+\infty)$ is a given continuous function so that $\lim _{t \rightarrow 0} f\left(t, x_{t}\right)=+\infty$ (i.e., $f$ is singular at $t=0$ ), where $C$ is the space of continuous functions from $[-\tau, 0]$ to $\mathbb{R}^{\geq 0}$ and $x_{t} \in C$ defined by $x_{t}(s)=x(t+s)$ for each $s \in[-\tau, 0)$. In the initial conditions of (1.1), we also assume that

$$
\text { (i) } x_{n-1} \geq 0, \quad \text { (ii) } x_{n-j} \geq \sum_{k=1}^{j-1} a_{k} x_{k+n-j}, \quad \forall j=2,3, \ldots, n-1 \text {. }
$$

The paper is organized as follows.

In Section 2, we provide some definitions about the fractional derivatives and the fractional integrals as well as we list their basic properties. Required topics of functional analysis such as Banach fixed point theorem and Leray-Schauder Theorem were also introduced. Section 3 deals with existence of a positive solution theorem and it gives an explainable example. The unique positive solution theorem with an explainable example has been discussed in Section 4.

\section{Preliminaries}

Preliminaries from fractional calculus [1, 4, 6] and functional analysis [17] are outlined below. 
Let $E$ be a real Banach space with a cone $K$. $K$ introduces a partial order $\leq$ in $E$ as $x \leq y$ if $y-x \in K$.

Definition 2.1. For $x, y \in E$, the order interval $\langle x, y\rangle$ is defined as

$$
\langle x, y\rangle=\{z \in E: x \leq z \leq y\}
$$

Theorem 2.2 (Leray-Schauder Theorem [17]). Let E be a Banach space with $C \subseteq E$ closed and convex. Assume $U$ is relatively open subset of $C$ with $0 \in U$ and $F: \bar{U} \rightarrow C$ is a continuous, compact map. Then, either

(i) F has fixed point in $\bar{U}$ or

(ii) there exists $u \in \partial U$ and $\gamma \in(0,1)$ with $u=\gamma F u$.

Theorem 2.3 (Banach fixed point theorem [17]). Let $K$ be a closed subspace of a Banach space $\Omega$. Let $F$ be a contraction mapping with Lipschitz constant $l(<1)$ from $K$ to itself. Then, $F$ has a unique fixed point $x^{*}$ in $K$. Moreover, if $x_{0}$ is an arbitrary point in $K$ and $\left\{x_{n}\right\}$ is defined by $x_{n+1}=F x_{n},(n=0,1,2, \ldots)$, then $\lim _{n \rightarrow \infty} x_{n}=x^{*} \in K$ and $d\left(x_{n}, x^{*}\right) \leq\left(k^{n} /(1-k)\right) d\left(x_{1}, x_{0}\right)$.

The complete Gamma function $\Gamma(\alpha)$ plays an important role in the theory of fractional integral and derivatives. A comprehensive definition of $\Gamma(\alpha)$ is provided by the Euler limit as

$$
\Gamma(\alpha)=\lim _{n \rightarrow \infty} \frac{n ! n^{\alpha}}{\alpha(\alpha+1) \cdots(\alpha+n)} .
$$

If $\alpha>0$, then $\Gamma(\alpha)$ has the following familiar integral representation:

$$
\Gamma(\alpha)=\int_{0}^{+\infty} t^{\alpha-1} e^{-t} d t
$$

In this paper, the Beta function $B(\alpha, \beta)$ is used. We notice that $B(\alpha, \beta)$ is closely related to the Gamma function. If $\alpha, \beta>0$, then it has the integral representation

$$
B(\alpha, \beta)=\int_{0}^{1} t^{\alpha-1}(1-t)^{\beta-1} d t=\frac{\Gamma(\alpha) \Gamma(\beta)}{\Gamma(\alpha+\beta)} .
$$

The definitions of Riemann-Liouville fractional derivative/integral and their properties are given bellow.

Definition 2.4. Let $x:[a, b] \rightarrow \mathbb{R}$ and $x \in L^{1}[a, b]$; then the expression

$$
I_{a^{+}}^{\alpha} x(t)=\frac{1}{\Gamma(\alpha)} \int_{a}^{t}(t-s)^{\alpha-1} x(t) d t, \quad x>a,
$$

is called a left-sided factional integrals of order $\alpha$. 
Definition 2.5. Let $n$ be a positive integer number and $\alpha \in(n-1, n]$. Then the left-sided fractional derivative of a function $x:[a, b] \rightarrow \mathbb{R}$ is defined as

$$
D_{a^{+}}^{\alpha} x(t)=D^{n}\left(I^{n-\alpha} x(t)\right), \quad t \in[a, b] .
$$

We denote $D_{a^{+}}^{\alpha} x(t)$ as $D_{a}^{\alpha} x(t)$ and $I_{a^{+}}^{\alpha} x(t)$ as $I_{a}^{\alpha} x(t)$. Further, $D^{\alpha} x(t)$ and $I^{\alpha} x(t)$ are referred as $D^{\alpha} x(t)$ and $I^{\alpha} x(t)$, respectively.

If the fractional derivative $D_{a}^{\beta} x(t)$ is integrable; then

$$
\begin{aligned}
I_{a}^{\alpha}\left(D_{a}^{\beta} x(t)\right)= & I_{a}^{\alpha-\beta} x(t)-\left\{I_{a}^{n-\beta} x(t)\right\}_{t=a} \frac{(t-a)^{\alpha-n}}{\Gamma(\alpha-n+1)} \\
& -\sum_{j=1}^{n-1}\left\{D_{a}^{\beta-j} x(t)\right\}_{t=a} \frac{(t-a)^{\alpha-j}}{\Gamma(\alpha-j+1)}
\end{aligned}
$$

where $n-1 \leq \beta<n, \beta \leq \alpha$, and $n \in \mathbb{N}[9]$. Further, if $x \in C[a, b]$, then $\left\{I_{a}^{n-\beta} x(t)\right\}_{t=a}=0$ and (1.4) reduces to

$$
I_{a}^{\alpha}\left(D_{a}^{\beta} x(t)\right)=I_{a}^{\alpha-\beta} x(t)-\sum_{j=1}^{n-1}\left\{D_{a}^{\beta-j} x(t)\right\}_{t=a} \frac{(t-a)^{\alpha-j}}{\Gamma(\alpha-j+1)} .
$$

\section{Existence Theorem}

In this section, we show that the initial value problem (1.1) under the conditions among the initial value (i.e., (1.3)) has a positive solution.

Lemma 3.1. Let $g:(0, b] \rightarrow \mathbb{R}$ be a continuous function and $\lim _{t \rightarrow 0^{+}} g(t)=+\infty$. If there exits $\sigma \in$ $(0,1)$ such that $\sigma<\alpha$ and by letting $t^{\sigma} g(t)$ be a continuous function on $[0, b]$, then $H(t)=I^{\alpha} t^{\sigma} g(t)$ is continuous $[0, b]$, where $n-1<\alpha<n, n \in \mathbb{N}$.

Proof. Let us consider $L=\max t^{\sigma} g(t), t \in[0, b]$. For all $t \in[0, b)$ and for given all $\epsilon>0$,

$$
\begin{aligned}
|H(t+\epsilon)-H(t)| & =\frac{1}{\Gamma(\alpha)}\left|\int_{0}^{t+\epsilon}(t+\epsilon-s)^{\alpha-1} d s-\int_{0}^{t}(t-s)^{\alpha-1} d s\right| \\
& =\frac{1}{\Gamma(\alpha)}\left|\int_{0}^{t+\epsilon}(t+\epsilon-s)^{\alpha-1} s^{-\sigma} s^{\sigma} g(s) d s-\int_{0}^{t}(t-s)^{\alpha-1} s^{-\sigma} s^{\sigma} g(s) d s\right| \\
& \leq \frac{L}{\Gamma(\alpha)}\left|\int_{0}^{t+\epsilon}(t+\epsilon-s)^{\alpha-1} s^{-\sigma} d s-\int_{0}^{t}(t-s)^{\alpha-1} s^{-\sigma} d s\right| \\
& =\frac{L}{\Gamma(\alpha)} B(1-\sigma, \alpha)\left|(t+\epsilon)^{\alpha-\sigma}-t^{\alpha-\sigma}\right| .
\end{aligned}
$$

Hence we conclude that $\lim _{\epsilon \rightarrow 0}|H(t+\epsilon)-H(t)|=0$. We notice that a similar result is conclusion for $|H(b-\epsilon)-H(b)|$. 
In the following, we want to show that (1.1) is equivalent to an integral equation.

Theorem 3.2. Suppose that $f:(0, b] \times C \rightarrow[0,+\infty)$ is a given continuous function so that $\lim _{t \rightarrow 0} f\left(t, x_{t}\right)=+\infty$ (i.e., $f$ is singular at $t=0$ ), where $C$ is the space of continuous functions from $[-\tau, 0]$ to $\mathbb{R}^{\geq 0}$ and $x_{t} \in C$ defined by $x_{t}(s)=x(t+s)$ for each $s \in[-\tau, 0]$. If there exists $\sigma \in(0,1)$ such that $0<\sigma<\alpha \in(n-1, n)$ and $t^{\sigma} f\left(t, x_{t}\right)$ is a continuous function on $[0, b]$, then the fractional differential equation

$$
\left(D^{\alpha}-\sum_{j=1}^{n-1} a_{j} D^{\alpha-j}\right)[x(t)-x(0)]=f\left(t, x_{t}\right), \quad t \in(0, b]
$$

is equivalent to the integral equation

$$
x(t)=x(0)+\lambda(t)+\mathcal{L}(I)[x(t)-x(0)]+I^{\alpha} f\left(t, x_{t}\right), \quad t \in[0, b],
$$

where

$$
\begin{gathered}
\lambda(t)=\sum_{j=1}^{n-1} \lambda_{j} t^{\alpha-j}, \quad \lambda_{j}=\frac{1}{\Gamma(\alpha-j+1)}\left(x_{j}-\sum_{k=1}^{n-j-1} a_{k} x_{k+j}\right), j=1, \ldots, n-2, \\
\lambda_{n-1}=\frac{1}{\Gamma(\alpha-n+1)}, \quad \mathcal{L}(I)=\sum_{j=1}^{n-1} a_{j} I^{j} x(t) .
\end{gathered}
$$

Proof. From (3.2), we have

$$
I^{\alpha}\left\{D^{\alpha}[x(t)-x(0)]\right\}-\sum_{j=1}^{n-1} a_{j} I^{\alpha}\left\{D^{\alpha-j}[x(t)-x(0)]\right\}=I^{\alpha} f\left(t, x_{t}\right), \quad t \in(0, b] .
$$

By using (2.7), we conclude that

$$
I^{\alpha}\left\{D^{\alpha}[x(t)-x(0)]\right\}=[x(t)-x(0)]-\sum_{j=1}^{n-1} \frac{x_{j} t^{\alpha-j}}{\Gamma(\alpha-j+1)},
$$

and for $k=1,2, \ldots, n-1$,

$$
I^{\alpha}\left\{D^{\alpha-k}[x(t)-x(0)]\right\}=I^{k}[x(t)-x(0)]-\sum_{j=1}^{n-k-1} \frac{x_{j+k} t^{\alpha-j}}{\Gamma(\alpha-j+1)}
$$

Note that, by Lemma 3.1, $I^{\alpha} f\left(t, x_{t}\right)=I^{\alpha}\left(t^{\sigma} t^{-\sigma} f\left(t, x_{t}\right)\right.$ exists and $D^{\alpha} I^{\alpha} f\left(t, x_{t}\right)=f\left(t, x_{t}\right)$, as $t^{-\sigma} f\left(t, x_{t}\right)$ is continuous and $I^{\alpha} f\left(t, x_{t}\right) \in C[0, b]$. In view of (3.5), (3.6), and (3.7), (3.2) is equivalent to the following integral equation:

$$
x(t)=x(0)+\lambda(t)+\mathcal{L}(I)[x(t)-x(0)]+I^{\alpha} f\left(t, x_{t}\right), \quad t \in(0, b],
$$


where

$$
\begin{gathered}
\lambda(t)=\sum_{j=1}^{n-1} \lambda_{j} t^{\alpha-j}, \quad \lambda_{j}=\frac{1}{\Gamma(\alpha-j+1)}\left(x_{j}-\sum_{k=1}^{n-j-1} a_{k} x_{k+j}\right), \quad j=1, \ldots, n-2, \\
\lambda_{n-1}=\frac{1}{\Gamma(\alpha-n+1)}, \quad \mathcal{L}(I)=\sum_{j=1}^{n-1} a_{j} I^{j} x(t) .
\end{gathered}
$$

The proof is completed. Therefore, by Theorem 3.2, the other expression of (1.1) is given as follows:

$$
x(t)= \begin{cases}x(0)+\mathcal{\lambda}(t)+\mathcal{L}(I)[x(t)-x(0)]+I^{\alpha} f\left(t, x_{t}\right), & t \in(0, b], \\ x(t)=\omega(t), & t \in[-\tau, 0]\end{cases}
$$

where $\lambda(t)$ and $\mathcal{L}(I)$ are mentioned in above.

Let $y:[-\tau, b] \rightarrow[0,+\infty)$ be function defined by

$$
y(t)= \begin{cases}\omega(0), & t \in[0, b] \\ \omega(t) \geq 0, & t \in[-\tau, 0]\end{cases}
$$

for each $z \in C([0, b], \mathbb{R})$ with $z(0)=0$; we denote by $\bar{z}$ the function define by

$$
\bar{z}(t)= \begin{cases}z(t), & t \in[0, b] \\ 0, & t \in[-\tau, 0]\end{cases}
$$

We can decompose $x(\cdot)$ as $x(t)=\bar{z}(t)+y(t), t \in[-\tau, b]$, which implies that $x_{t}=\bar{z}_{t}+y_{t}, t \in$ $[0, b]$. Hence by Theorem 3.2, (1.1) is equivalent to the following integral equation:

$$
z(t)=\lambda(t)+\mathcal{L}(I) z(t)+I^{\alpha} f\left(t, \bar{z}_{t}+y_{t}\right), \quad t \in[0, b] .
$$

Set $\Omega=\{z \in C([0, b], \mathbb{R}), z(0)=0\}$, and for each $z \in \Omega$, let $\|z\|_{b}$ be the seminorm in $\Omega$ defined by

$$
\|z\|_{b}=\|z(0)\|+\|z\|=\|z\|=\sup \{|z(t)|: t \in[0, b]\} .
$$

$\Omega$ is a Banach space with norm $\|\cdot\|_{b}$. Let $K$ be a cone of $\Omega . K=\{z \in \Omega: z(t) \geq 0, t \in[0, b]\}$ and

$$
K^{*}=\left\{x \in C\left([-\tau, b], \mathbb{R}^{\geq 0}\right): x(t)=\omega(t) \geq 0, \quad t \in[-\tau, 0]\right\} .
$$

Define the operator $F: K \rightarrow K$ by 


$$
F z(t)=\lambda(t)+\mathcal{L}(I) z(t)+I^{\alpha} f\left(t, \bar{z}_{t}+y_{t}\right), \quad t \in[0, b] .
$$

Theorem 3.3. Suppose that $f\left(t, x_{t}\right), t \in(0, b], x_{t} \in C$, is a continuous function and $\lim _{t \rightarrow 0^{+}} f(t, \cdot)=+\infty$. If there exits $\sigma \in(0,1)$ such that $0<\sigma<\alpha \in(n-1, n)$ and $t^{\sigma} f\left(t, x_{t}\right)$ 庭 a continuous function on $[0, b]$, then the operator $F$, defined as (3.12), maps bounded set into bounded sets in $K$, continuous and completely continuous.

Proof. For all $u \in K$, since $F u(t)=\lambda(t)+\mathcal{L}(I) u(t)+I^{\alpha} f\left(t, \bar{u}_{t}+y_{t}\right)$ by Lemma 3.1 and the nonnegativeness of $f$, we obtain $F: K \rightarrow K$.

Since $t^{\sigma} f\left(t, x_{t}\right)$ is continuous on $[0, b] \times[0,+\infty)$, there exists a positive constant $N$ such that $t^{\sigma} f\left(t, x_{t}\right) \leq N$. Hence

$$
\begin{aligned}
I^{\alpha} f\left(t, \bar{z}_{t}+y_{t}\right) & =I^{\alpha}\left(t^{-\sigma} t^{\sigma} f\left(t, x_{t}\right)\right) \\
& \leq N I^{\alpha} t^{-\sigma}=\frac{N}{\Gamma(\alpha)} \int_{0}^{t}(t-s)^{\alpha-1} s^{-\sigma} d s \\
& =\frac{N t^{\alpha-\sigma}}{\Gamma(\alpha)} B(1-\sigma, \alpha) \leq \frac{N b^{\alpha-\sigma}}{\Gamma(\alpha)} B(1-\sigma, \alpha) .
\end{aligned}
$$

Let $G \subset K$ be bounded, that is, there exists a positive constant $L$ such that $\|z\|_{b} \leq L$, for all $z \in C$. In view of (3.13), for each $z \in G$, we obtain

$$
\begin{aligned}
\|F z(t)\| & \leq|\lambda(t)|+|\mathcal{L}(I) z(t)|+\left|I^{\alpha} f\left(t, \bar{z}_{t}+y_{t}\right)\right| \\
& \leq\|\lambda(t)\|+\|z\|_{b} \sum_{j=1}^{n} \frac{a_{j} b^{j}}{\Gamma(j+1)}+\frac{N b^{\alpha-\sigma}}{\Gamma(\alpha)} B(1-\sigma, \alpha) \\
& \leq\|\lambda(t)\|+L \sum_{j=1}^{n} \frac{a_{j} b^{j}}{\Gamma(j+1)}+\frac{N b^{\alpha-\sigma}}{\Gamma(\alpha)} B(1-\sigma, \alpha) .
\end{aligned}
$$

Thus, $F(K)$ is bounded. In the following text, we would like to show that $F$ is continuous. Let $v_{0} \in K$ and $\left\|v_{0}\right\|=c_{0}$, if $v \in K$ and $\left\|v-v_{0}\right\|<l$, then $\|v\|<l+c_{0}$; by the continuity of $t^{\sigma} f\left(t, \bar{z}_{t}+y_{t}\right)$, we know that $t^{\sigma} f\left(t, \bar{z}_{t}+y_{t}\right)$ is uniformly continuous on $[0, b] \times[0, c]$. Thus, for all $\epsilon>0$ there exists a $\delta>0$ such that

$$
\left|t^{\sigma} f\left(t, \bar{u}_{t}+y_{t}\right)-t^{\sigma} f\left(t, \bar{v}_{t}+y_{t}\right)\right|<\epsilon
$$

for all $t \in[0, b]$ and $u, v \in[0, c]$ with $|u-v|<\delta$. Obviously, if $\|u-v\|<\delta$, then $v_{0}(t), v(t) \in$ $[0, c]$ and $\left|v(t)-v_{0}(t)\right|<\delta$ for each $t \in[0, b]$. Hence, we have

$$
\left|t^{\sigma} f\left(t, \bar{v}_{t}+y_{t}\right)-t^{\sigma} f\left(t, \bar{v}_{0}+y_{t}\right)\right|<\epsilon
$$

for all $t \in[0, b]$ and $v \in K$ with $\left\|v-v_{0}\right\|<\delta$. For all $t \in[0, b]$, let $u, v \in K$, and $|u(t)-v(t)|<\delta$, we choose $\delta \leq \epsilon\left(\sum_{j=1}^{n-1}\left(x_{j} b^{j} / \Gamma(j+1)\right)\right)^{-1}$. By using (3.20), we get 


$$
\begin{aligned}
|F u(t)-F v(t)| \leq & \|u-v\|_{b} \sum_{j=1}^{n-1} \frac{a_{j} b^{j}}{\Gamma(j+1)}+\left|I^{\alpha}\left(f\left(t, \bar{u}_{t}+y_{t}\right)-f\left(t, \bar{v}_{t}+y_{t}\right)\right)\right| \\
= & \|u-v\|_{b} \sum_{j=1}^{n-1} \frac{a_{j} b^{j}}{\Gamma(j+1)}+\left|I^{\alpha}\left(t^{-\sigma} t^{\sigma} f\left(t, \bar{u}_{t}+y_{t}\right)-t^{-\sigma} t^{\sigma} f\left(t, \bar{v}_{t}+y_{t}\right)\right)\right| \\
\leq & \|u-v\|_{b} \sum_{j=1}^{n-1} \frac{a_{j} b^{j}}{\Gamma(j+1)} \\
& +\frac{1}{\Gamma(\alpha)} \max _{0 \leq t \leq b} \int_{0}^{t}(t-s)^{\alpha-1} s^{-\sigma}\left|s^{\sigma} f\left(t, \bar{u}_{t}+y_{t}\right)-s^{\sigma} f\left(t, \bar{v}_{t}+y_{t}\right)\right| d s \\
\leq & \|u-v\|_{b} \sum_{j=1}^{n-1} \frac{a_{j} b^{j}}{\Gamma(j+1)}+\frac{\epsilon}{\Gamma(\alpha)} \max _{0 \leq t \leq b} \int_{0}^{t}(t-s)^{\alpha-1} s^{-\sigma} d s \\
= & \|u-v\|_{b} \sum_{j=1}^{n-1} \frac{a_{j} b^{j}}{\Gamma(j+1)}+\frac{\epsilon}{\Gamma(\alpha)} \max _{0 \leq t \leq b}^{\alpha-\sigma} B(1-\sigma, \alpha) \\
\leq & \left(1+b^{\alpha-\sigma}\right) B(1-\sigma, \alpha) \epsilon .
\end{aligned}
$$

Finally, we want to prove that the operator $F$ is equicontinuous. Let $G \subseteq K$ be bounded, that is, three exits a positive constant $l$ such that $\|u\| \leq l$, for all $u \in G$. Suppose, $u \in K, t, r \in[0, b]$ and $t<r$. For a given $\epsilon>0$, there exists $\delta>0$, so that if $|t-r|<\delta$, then

$$
\begin{aligned}
\mid F u(t) & -F u(r) \mid \\
\leq & |\lambda(t)-\lambda(r)|+|\mathcal{L}(I) u(t)-\mathcal{L}(I) u(r)|+\left|I^{\alpha} f\left(t, \bar{u}_{t}+y_{t}\right)-I^{\alpha} f\left(r, \bar{u}_{r}+y_{r}\right)\right| \\
\leq & \sum_{j=1}^{n-1} \lambda_{j}\left|t^{\alpha-j}-r^{\alpha-j}\right|+\sum_{j=1}^{n-1} \frac{l a_{j}}{\Gamma(j)}\left|\int_{0}^{t}(t-s)^{j-1}-(r-s)^{j-1} d s\right| \\
& +\sum_{j=1}^{n-1} \frac{l a_{j}}{\Gamma(j)} \int_{t}^{r}(r-s)^{j-1} d s \\
& +\frac{1}{\Gamma(\alpha)}\left|\int_{0}^{t}(t-s)^{\alpha-1} s^{-\sigma} s^{\sigma} f\left(s, \bar{u}_{s}+y_{s}\right) d s-\int_{0}^{r}(r-s)^{\alpha-1} s^{-\sigma} s^{\sigma} f\left(s, \bar{u}_{s}+y_{s}\right) d s\right| \\
\leq & 2 \sum_{j=1}^{n-1} \lambda_{j} b^{\alpha-j}+l \sum_{j=1}^{n-1} \frac{a_{j} t^{j}}{\Gamma(j+1)}+l \sum_{j=1}^{n-1} \frac{a_{j}}{\Gamma(j+1)}\left[|r-t|^{j}+\left|r^{j}-t^{j}\right|\right] \\
& +\frac{N B(1-\sigma, \alpha)}{\Gamma(\alpha)}\left|t^{\alpha-\sigma}-r^{\alpha-\sigma}\right|
\end{aligned}
$$


Abstract and Applied Analysis

$$
\begin{aligned}
\leq & 2 \sum_{j=1}^{n-1} \lambda_{j} b^{\alpha-j}+l \sum_{j=1}^{n-1} \frac{a_{j} b^{j}}{\Gamma(j+1)}+2 l \sum_{j=1}^{n-1} \frac{a_{j}}{\Gamma(j+1)}+l \sum_{j=1}^{n-1} \frac{a_{j}}{\Gamma(j+1)}|r-t|^{j} \\
& +\frac{2 N B(1-\sigma, \alpha)}{\Gamma(\alpha)} b^{\alpha-\sigma} .
\end{aligned}
$$

Set $\Delta=\max \left\{3 \Delta_{1} / \epsilon, 3 \Delta_{2} / \epsilon, \Delta_{3}, \Delta_{4}, \ldots, \Delta_{n+1}\right\}$, where

$$
\begin{gathered}
\Delta_{1}=\sum_{j=1}^{n-1}\left(2 \lambda_{j} b^{\alpha-j}+\frac{l a_{j} b^{j}}{\Gamma(j+1)}+\frac{2 l a_{j}}{\Gamma(j+1)}\right) \\
\Delta_{2}=\frac{2 N}{\Gamma(\alpha)} B(1-\sigma, \alpha) b^{\alpha-\sigma}, \\
\Delta_{j+2}=l \sum_{j=1}^{n-1} \frac{a_{j}}{\Gamma(j+1)}, \quad j=1,2, \ldots, n-1 .
\end{gathered}
$$

Case 1. If $0<|r-t|<1<b$, we choose $\delta=\epsilon /(3(n-1))$. Hence,

$$
\begin{aligned}
|F u(t)-F u(r)| & \leq \frac{\epsilon}{3} \Delta+\frac{\epsilon}{3} \Delta+\Delta \sum_{j=1}^{n-1}|r-t|^{j} \\
& \leq \frac{\epsilon}{3} \Delta+\frac{\epsilon}{3} \Delta+(n-1) \Delta \delta<\Delta \epsilon .
\end{aligned}
$$

Case 2. If $0<1 \leq|r-t|<b$, we choose $\delta=\{\epsilon /(3(n-1))\}^{1 /(n-1)}$. Hence,

$$
\begin{aligned}
|F u(t)-F u(r)| & \leq \frac{\epsilon}{3} \Delta+\frac{\epsilon}{3} \Delta+\Delta \sum_{j=1}^{n-1}|r-t|^{j} \\
& \leq \frac{\epsilon}{3} \Delta+\frac{\epsilon}{3} \Delta+(n-1) \Delta \delta^{n-1}<\Delta \epsilon .
\end{aligned}
$$

Therefore, $F(G)$ is equicontinuous. The Arzela-Ascoli Theorem implies that $\overline{F(G)}$ is compact. Thus, $F: K \rightarrow K$ is completely continuous.

Theorem 3.4. With all the details of (1.1)-(1.3), further, if $\sigma$ exists in $(0,1)$ such that $0<\sigma<\alpha \in$ $(n-1, n), n \in \mathbb{N}$, and $t^{\sigma} f\left(t, x_{t}\right)$ is a continuous function on $[0, b]$, then (1.1) has at least one positive solution $x^{*} \in K^{*}$, satisfying $\left\|x^{*}\right\| \leq \max \{\|\omega\|, h\}$, where $h=2 \Lambda /(1-\Lambda)+1$ and $\Lambda$ is a positive constant which is observed in the proof of the theorem.

Proof. We know that the operator $F: K \rightarrow K$ is continuous and it is completely continuous by Theorem 3.3. Here we show that there exists an open set $U \subseteq K$, with $z \neq \gamma F(z)$ for $\gamma \in(0,1)$ 
and $z \in \partial U$. Let $z \in K$ be any solution of $z=\gamma F(z), \gamma \in(0,1)$. According to the Theorem 3.3, since $F: K \rightarrow K$ is continuous and it is completely continuous, we have

$$
\begin{aligned}
z(t) & =\gamma F z(t)=\gamma\left\{\lambda(t)+\mathcal{L}(I) z(t)+I^{\alpha} f\left(t, \bar{z}_{t}+y_{t}\right)\right\}, \quad t \in[0, b] \\
& \leq \sum_{j=1}^{n-1} \lambda_{j} b^{j}+\sum_{j=1}^{n-1} a_{j}\left|I^{j} z(t)\right|+\left|I^{\alpha} f\left(t, \bar{z}_{t}+y_{t}\right)\right| \\
& \leq \sum_{j=1}^{n-1} \lambda_{j} b^{j}+\|z\| \sum_{j=1}^{n-1} \frac{a_{j}}{\Gamma(j+1)} b^{j}+\frac{1}{\Gamma(\alpha)}\left|\int_{0}^{t}(t-s)^{\alpha-1} s^{-\sigma} s^{\sigma} f\left(s, \bar{z}_{s}+y_{s}\right) d s\right| .
\end{aligned}
$$

Since there exists a positive constant $N$ such that $\left\|s^{\sigma} f\left(s, \bar{z}_{s}+y_{s}\right)\right\| \leq N$, as $s^{\sigma} f\left(s, \bar{z}_{s}+y_{s}\right)$ is continuous on $[0, b]$, therefore

$$
z(t) \leq \sum_{j=1}^{n-1} \lambda_{j} b^{j}+\|z\| \sum_{j=1}^{n-1} \frac{a_{j}}{\Gamma(j+1)} b^{j}+\frac{N}{\Gamma(\alpha)} B(1-\sigma, \alpha)
$$

Set $\Lambda=\max \left\{\Lambda_{1}, \Lambda_{2}, \Lambda_{3}\right\}$, where

$$
\Lambda_{1}=\sum_{j=1}^{n-1} \lambda_{j} b^{j}, \quad \Lambda_{2}=\sum_{j=1}^{n-1} \frac{a_{j}}{\Gamma(j+1)} b^{j}, \quad \Lambda_{3}=\frac{N}{\Gamma(\alpha)} B(1-\sigma, \alpha) .
$$

Equation (3.20) implies that

$$
\|z\| \leq \Lambda_{1}+\|z\| \Lambda_{2}+\Lambda_{3} \leq 2 \Lambda+\|z\| \Lambda .
$$

Therefore, we conclude that $\|z\|(1-\Lambda) \leq 2 \Lambda$. Then, as a result, any solution $z=\gamma F(z)$ satisfies $\|z\| \neq h$. Let $U=\{z \in K:\|z\|<h\}$. Theorem 2.2 guarantees that $F$ has a fixed point $z \in \bar{U}$. By using Theorem 3.2, (1.1) under the conditions of (1.3) has a positive solution $x^{*} \in K$ satisfying $\left\|x^{*}\right\| \leq \max \{\|\omega\|, h\}$.

Example 3.5. Consider the following nonlinear fractional differential equation:

$$
\begin{gathered}
\mathcal{L}(D)[x(t)-x(0)]=t^{-1 / 2} x_{t}, \quad t \in(0,1], \\
x(t)=-\sin t \geq 0, \quad t \in[-1,0],
\end{gathered}
$$

where $\mathcal{L}(D)=D^{5 / 2}-D^{3 / 2}-D^{1 / 2}$. Here, $a_{1}=a_{2}=1, \alpha=5 / 2 \in(2,3), n=3$ and $f\left(t, x_{t}\right)=$ $t^{-1 / 2} x_{t}$ such that $\lim _{t \rightarrow 0^{+}} f(t, \cdot)=+\infty$. We select $\sigma=11 / 12,0<\sigma<\alpha$. Hence $t^{\sigma} f(t, \cdot)=t^{5 / 12} x_{t}$ is continuous on $[0,1]$. We consider the initial conditions

$$
x(0)=0, \quad x_{1}=\left.D^{3 / 2} x(t)\right|_{t=0}=2, \quad x_{2}=\left.D^{5 / 2} x(t)\right|_{t=0}=1, \quad x_{1} \geq x_{2}>0 .
$$


By using Theorem 3.2, the nonlinear fractional differential equation (3.20) is equivalent to an integral equation given below

$$
x(t)= \begin{cases}\lambda(t)+\mathcal{L}(I) x(t)=I^{5 / 2}\left(t^{-1 / 2} x_{t}\right), & t \in(0,1], \\ x(t)=-\sin \pi t, & t \in[-1,0],\end{cases}
$$

where in the view of the procedure proving Theorem 3.2, we have

$$
\begin{aligned}
& \lambda(t)=\sum_{j=1}^{2} \lambda_{j} t^{5 / 2-j}=\frac{1}{\Gamma(5 / 2)} t^{3 / 2}+\frac{1}{\Gamma(1 / 2)} t^{1 / 2}, \\
& \mathcal{L}(I) x(t)=\sum_{j=1}^{2} a_{j} I^{j} x(t)=\int_{0}^{t}(1+(t-s)) x(s) d s .
\end{aligned}
$$

In addition of that we have that $\Lambda=\max \left\{\Lambda_{1}, \Lambda_{2}, \Lambda_{3}\right\}$, where

$$
\begin{gathered}
\Lambda_{1}=\sum_{j=1}^{2} \lambda_{j} b^{j}=\lambda_{1}+\lambda_{2}=\frac{1}{\Gamma(1 / 2)}+\frac{1}{\Gamma(5 / 2)}=\frac{7}{3 \sqrt{\pi}}, \\
\Lambda_{2}=\sum_{j=1}^{2} \frac{a_{j}}{\Gamma(j+1)}=1+\frac{1}{2}=\frac{3}{2}, \\
\Lambda_{3}=\frac{N}{\Gamma 52} B\left(1-\frac{11}{12}, \frac{5}{2}\right)=\frac{4 N}{3 \sqrt{\pi}} B\left(\frac{1}{12}, \frac{5}{2}\right), \quad N=\left\|t^{11 / 12} x_{t}\right\| .
\end{gathered}
$$

Therefore, by using the Theorem 3.4, (3.30) has at least one positive solution $x^{*} \in K^{*}$ satisfying $\left\|x^{*}\right\| \leq \max \{\|\omega\|, h\}$ where $\|\omega\|=\max _{-1 \leq t \leq 0}|-\sin \pi t|=1$ and $h=\Lambda /(1-\Lambda)+1, \Lambda=$ $\max \left\{\Lambda_{1}, \Lambda_{2}, \Lambda_{3}\right\}$.

\section{Unique Existence of Solution}

In this section, we give conditions on $f$ and $a_{1}, a_{2}, \ldots, a_{n}$, which render a unique positive solution to (1.1).

Theorem 4.1. Let $f:(0,1) \times[0, \infty) \rightarrow[0, \infty)$ be continuous and $\lim _{t \rightarrow 0^{+}} f(t, \cdot)=+\infty$. Suppose that there exists $\sigma \in(0,1)$ so that $0<\sigma<\alpha \in(n-1, n), n \in \mathbb{N}$, and $t^{\sigma} f\left(t, x_{t}\right)$ is a continuous function on $[0, b]$. If further, the following conditions are satisfied

(H1) $t^{\sigma} f\left(t, x_{t}\right)$ is Lipschitz with respect to the second variable with Lipschitz constant $\mu$, that is,

$$
\left|t^{\sigma} f\left(t, x_{t}\right)-t^{\sigma} f\left(t, z_{t}\right)\right| \leq \mu\|\bar{u}-\bar{v}\|, \quad \forall u, v \in K, t \in(0, b]
$$


(H2)

$$
0<\sum_{j=1}^{n-1} \frac{a_{j} b^{j}}{\Gamma(j+1)}+\frac{\mu b^{\alpha-\sigma}}{\Gamma(\alpha)} B(1-\sigma, \alpha)<1
$$

where $x(t)=u(t)+y(t)$ and $z(t)=v(t)+y(t)$; then (1.1) under the conditions of (1.3) has a unique positive solution.

Proof. As it was pointed out in the previous section, (1.1) is equivalent to (3.13). Thus, for $u, v \in K$ we obtain

$$
\begin{aligned}
|F u(t)-F v(t)|= & |\mathcal{L}(I) u(t)-\mathcal{L}(I) v(r)|+\left|I^{\alpha} f\left(t, \bar{u}_{t}+y_{t}\right)-I^{\alpha} f\left(r, \bar{v}_{r}+y_{r}\right)\right| \\
\leq & \|u-v\|_{b} \sum_{j=1}^{n-1} \frac{a_{j} b^{j}}{\Gamma(j+1)}+\left|I^{\alpha}\left(f\left(t, \bar{u}_{t}+y_{t}\right)-f\left(t, \bar{v}_{t}+y_{t}\right)\right)\right| \\
= & \|u-v\|_{b} \sum_{j=1}^{n-1} \frac{a_{j} b^{j}}{\Gamma(j+1)}+\left|I^{\alpha}\left(t^{-\sigma} t^{\sigma} f\left(t, \bar{u}_{t}+y_{t}\right)-t^{-\sigma} t^{\sigma} f\left(t, \bar{v}_{t}+y_{t}\right)\right)\right| \\
\leq & \|u-v\|_{b} \sum_{j=1}^{n-1} \frac{a_{j} b^{j}}{\Gamma(j+1)} \\
& +\frac{1}{\Gamma(\alpha)} \max _{0 \leq t \leq b} \int_{0}^{t}(t-s)^{\alpha-1} s^{-\sigma}\left|s^{\sigma} f\left(t, \bar{u}_{t}+y_{t}\right)-s^{\sigma} f\left(t, \bar{v}_{t}+y_{t}\right)\right| d s \\
\leq & \|u-v\|_{b} \sum_{j=1}^{n-1} \frac{a_{j} b^{j}}{\Gamma(j+1)}+\frac{\mu}{\Gamma(\alpha)} \max _{0 \leq t \leq b} \int_{0}^{t}(t-s)^{\alpha-1} s^{-\sigma} d s \\
\leq & \|u-v\|_{b} \sum_{j=1}^{n-1} \frac{a_{j} b^{j}}{\Gamma(j+1)}+\frac{\mu\|u-v\|_{b}}{\Gamma(\alpha)} \max _{0 \leq t \leq b} t^{\alpha-\sigma} B(1-\sigma, \alpha) \\
\leq & \|u-v\|_{b}\left\{\sum_{j=1}^{n-1} \frac{a_{j} b^{j}}{\Gamma(j+1)}+\frac{\mu b^{\alpha-\sigma}}{\Gamma(\alpha)} B(1-\sigma, \alpha)\right.
\end{aligned},
$$

where $F$ is given in (3.16). Hence, in view of the Theorem 2.3, $F$ will have a unique fixed point in $K$, which is the unique positive solution of (1.1).

Example 4.2. Consider the nonlinear fractional differential equation given below

$$
\begin{gathered}
\mathcal{L}(D)[x(t)-x(0)]=\frac{\eta}{\sqrt{t}} \int_{-\pi}^{-t} \frac{1+x_{t}(s)}{1+x_{t}^{2}(s)} d s, \quad t \in(0, \pi], \eta>0, \\
x(t)=-\sin t \geq 0, \quad t \in[-\pi, 0],
\end{gathered}
$$

where $\mathcal{L}(D)=D^{5 / 2}-a_{1} D^{3 / 2}-a_{2} D^{1 / 2}$ with initial conditions $x(0)=0, x_{1}=\left.D^{3 / 2} x(t)\right|_{t=0}=$ $2, x_{2}=\left.D^{5 / 2} x(t)\right|_{t=0}=1, x_{1} \geq x_{2}>0,0<a_{1} \leq 1 /(4 \pi), 0<a_{2} \leq 8 / \pi^{2}$, and 
$\eta=3 \pi^{-3 / 7)} / 16 B(1 / 12,5 / 12)$. Here $\lim _{t \rightarrow 0^{+}} f\left(t, x_{t}\right)=+\infty$. We select $\sigma=11 / 12$; then $0<\sigma<\alpha$ and $t^{\sigma} f\left(t, x_{t}\right)=t^{5 / 12} f\left(t, x_{t}\right)$ is continuous on $[0, \pi]$. Now, we review the Lipschitz condition with respect to the second variable. Note that from equations (3.11) and (3.12), for $x_{t}, z_{t} \in C\left([-\pi, 0], \mathbb{R}^{\geq 0}\right)$, we have $x_{t}=\bar{u}_{t}+y_{t}, z_{t}=\bar{v}_{t}+y_{t}, t \in[-\pi, \pi]$ and $\left.x_{t}=u(t)+y(t), \quad z_{t}=v(t)+y(t), t \in(0, \pi]\right)$ where $u, v \in K$. Without loss of generality, we suppose that $x_{t}(s) \geq y_{t}(s)$, for all $s \in[-\pi, 0]$. Then, for each $t \in(0, \pi]$,

$$
\begin{aligned}
\left|t^{11 / 12} f\left(t, x_{t}\right)-t^{11 / 12} f\left(t, z_{t}\right)\right| & =\eta t^{11 / 12} \int_{-\pi}^{-t}\left|\frac{1+x(t+s)}{1+x^{2}(t+s)}-\frac{1+z(t+s)}{1+z^{2}(t+s)}\right| d s \\
& =\eta t^{11 / 12} \int_{t-\pi}^{0}\left|\frac{1+x(s)}{1+x^{2}(s)}-\frac{1+z(s)}{1+z^{2}(s)}\right| d s \\
& \leq \eta t^{11 / 12} \int_{t-\pi}^{0}\left|\frac{1+x(s)}{1+x^{2}(s)}-\frac{1+z(s)}{1+x^{2}(s)}\right| d s \\
& \leq \eta \max _{0 \leq t \leq \pi}^{11 / 12} \int_{t-\pi}^{0} \frac{|x(s)-z(s)|}{1+x^{2}(s)} d s \\
& \leq \eta\|x(t)-z(t)\| \max _{0 \leq t \leq \pi} t^{11 / 12} \int_{t-\pi}^{0} d s \\
& =\eta\|u-v\| \max _{0 \leq t \leq \pi} t^{11 / 12}(\pi-t)=\eta \pi^{23 / 12}\|u-v\| .
\end{aligned}
$$

Hence, the condition of (H1) holds with $\mu=\pi^{23 / 12} \eta$. On other hand, we have

$$
\frac{a_{1} b}{\Gamma(2)}+\frac{a_{2} b^{2}}{\Gamma(3)}+\frac{\mu \pi^{11 / 12}}{\Gamma(5 / 2)} B\left(\frac{1}{12}, \frac{5}{12}\right) \in(0,1)
$$

Thus, (4.4) satisfies the conditions required by the Theorem 4.1. This Theorem implies that the nonlinear equation (4.4) has a unique solution in $K$. By using the Theorem 3.2, (4.4) is equivalent to the following integral equation:

$$
x(t)=\frac{1}{\Gamma(5 / 2)}\left(2-\frac{1}{4 \pi}\right) t^{3 / 2}+\frac{1}{\Gamma(3 / 2)} t^{1 / 2}+a_{1} I x(t)+a_{2} I^{2} x(t)+I^{5 / 2} f\left(t, x_{t}\right) .
$$


The solution of $(4.4)$ is $x(t)=\lim _{n \rightarrow+\infty} x_{n}(\mathrm{t})$, where $x_{n+1}(t)=F x_{n}(t)$. Therefore, the iterated sequence is given by

$$
\begin{aligned}
x_{1}(t) & =a_{1} t^{3 / 2}+a_{2} t^{1 / 2}+\frac{\eta e^{-t}}{\sqrt{t}} I^{5 / 2}\left[\int_{-\pi}^{-t} \frac{1+x(s)}{1+x^{2}(s)} d s\right] \\
& =a_{1} t^{3 / 2}+a_{2} t^{1 / 2}+\frac{\eta e^{-t}}{\sqrt{t}} I^{5 / 2}\left[\frac{1}{2} \ln \left(\frac{1+t^{2}}{1+\pi^{2}}\right)-\tan ^{-1} t\right], \\
x_{2}(t) & =\left(a_{1} I+a_{2} I^{2}+I^{5 / 2}\right) x_{1}(t)+x_{1}(t), \\
& \vdots \\
x_{n+1}(t) & =\sum_{i=0}^{n}\left(a_{1} I+a_{2} I^{2}+I^{5 / 2}\right)^{n-i} x_{1}(t), \quad n=1,2, \ldots
\end{aligned}
$$

\section{References}

[1] I. Podlubny, Fractional Differential Equations, vol. 198 of Mathematics in Science and Engineering, Academic Press, San Diego, Calif, USA, 1999.

[2] K. B. Miller and B. Ross, An Introduction to the Fractional Calculus and Fractional Differential Equations, Wiely, New York, NY, USA, 1993.

[3] K. B. Oldham and I. Spanier, The Fractional Caculus, Academic Press, London, UK, 1997.

4. S. G. Samko, A. A. Kilbas, and O. I. Marichev, Fractional Integrals and Derivatives, Gordon and Breach Science Publishers, Yverdon, Switzerland, 1993.

[5] B. J. West, M. Bologna, and P. Grigolini, Eds., Physics of Fractal Operators, Institute for Nonlinear Science, Springer, New York, NY, USA, 2003.

[6] A. A. Kilbas, H. M. Srivastava, and J. J. Trujillo, Theory and Applications of Fractional Differential Equations, vol. 204 of North-Holland Mathematics Studies, Elsevier Science B.V., Amsterdam, The Netherlands, 2006.

[7] R. L. Magin, Fractional Calculus in Bioengineering, Begell House Publisher, Redding, Conn, USA, 2006.

8. A. Babakhani and E. Enteghami, "Existence of positive solutions for multiterm fractional differential equations of finite delay with polynomial coefficients," Abstract and Applied Analysis, vol. 2009, Article ID 768920, 12 pages, 2009.

[9] V. Daftardar-Gejji and A. Babakhani, "Analysis of a system of fractional differential equations," Journal of Mathematical Analysis and Applications, vol. 293, no. 2, pp. 511-522, 2004.

[10] A. Babakhani and V. Daftardar-Gejji, "Existence of positive solutions of nonlinear fractional differential equations," Journal of Mathematical Analysis and Applications, vol. 278, no. 2, pp. 434-442, 2003.

[11] A. Babakhani and V. Daftardar-Gejji, "Existence of positive solutions for multi-term non-autonomous fractional differential equations with polynomial coefficients," Electronic Journal of Differential Equations, no. 129, pp. 1-12, 2006.

[12] C. Sign and B. Chuanzhi, "Positive solutions for nonlinear fractional differential equations with coefficient that changes sign," Nonlinear Analysis. Theory, Methods \& Applications, vol. 64, no. 4, pp. 677-685, 2006.

[13] V. Daftardar-Gejji, "Positive solutions of a system of non-autonomous fractional differential equations," Journal of Mathematical Analysis and Applications, vol. 302, no. 1, pp. 56-64, 2005.

[14] H. Ye, Y. Ding, and J. Gao, "The existence of a positive solution of a fractional differential equation with delay," Positivity, vol. 11, no. 2, pp. 341-350, 2007.

[15] X. Zhong," Some results of linear order fractional order time-delay system," Applied Mathematics and Application, vol. 197, pp. 407-411, 2007.

[16] A. Belarbi, M. Benchohra, and A. Ouahab, “Uniqueness results for fractional functional differential equations with infinite delay in Fréchet spaces," Applicable Analysis, vol. 85, no. 12, pp. 1459-1470, 2006.

[17] M. C. Joshi and R. K. Bose, Some Topics in Nonlinear Functional Analysis, John Wiley \& Sons, New York, NY, USA, 1985. 


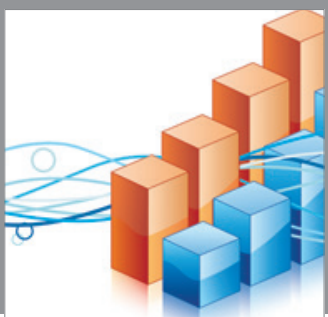

Advances in

Operations Research

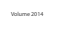

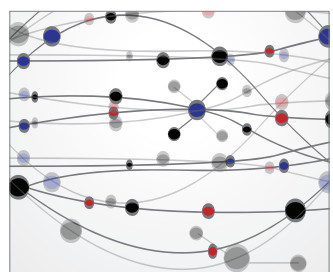

\section{The Scientific} World Journal
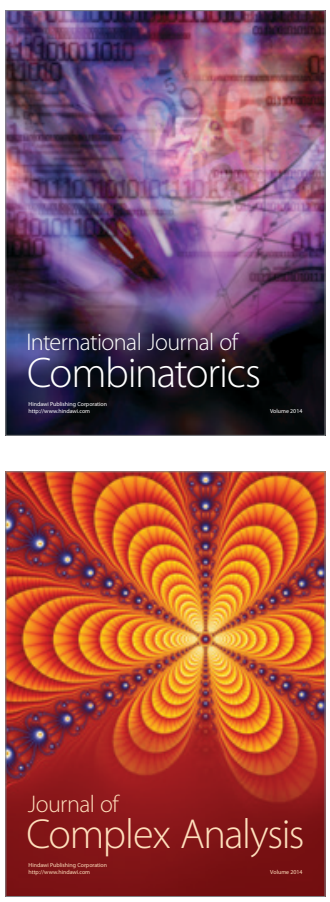

International Journal of

Mathematics and

Mathematical

Sciences
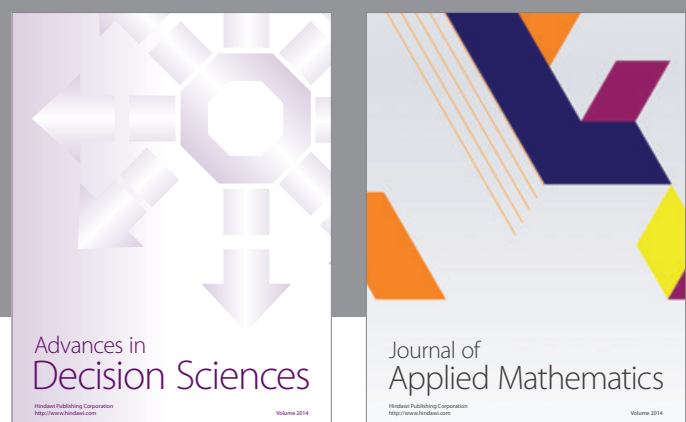

Journal of

Applied Mathematics
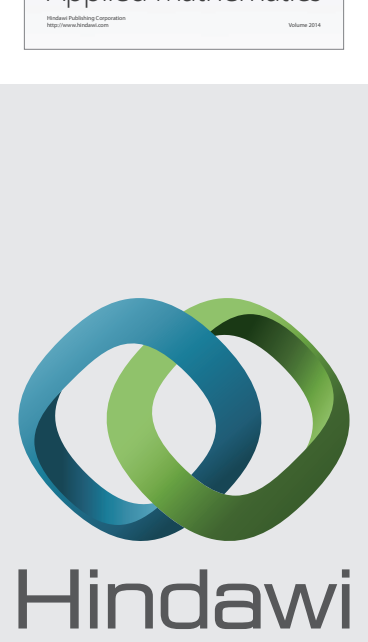

Submit your manuscripts at http://www.hindawi.com
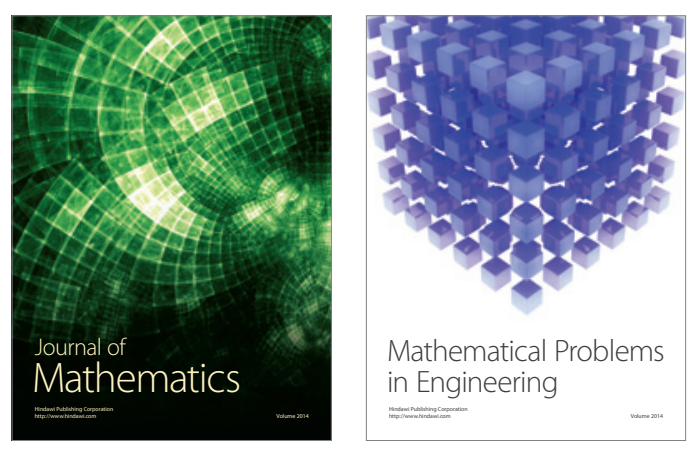

Mathematical Problems in Engineering
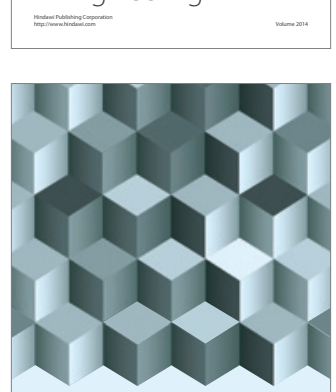

Journal of

Function Spaces
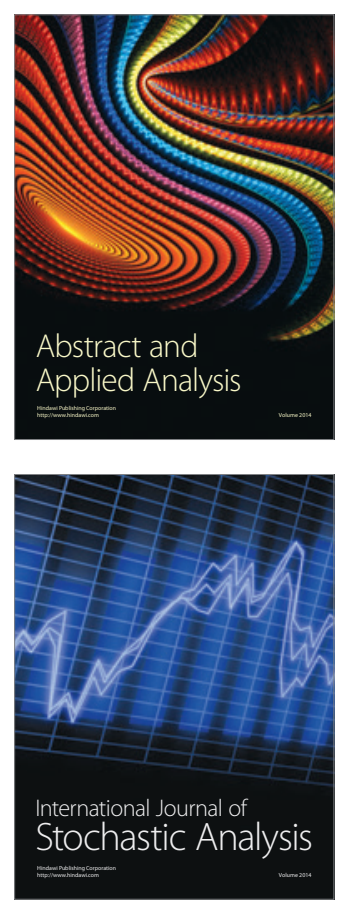

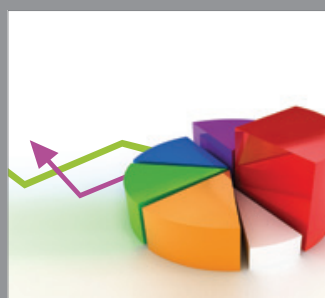

ournal of

Probability and Statistics

Promensencen
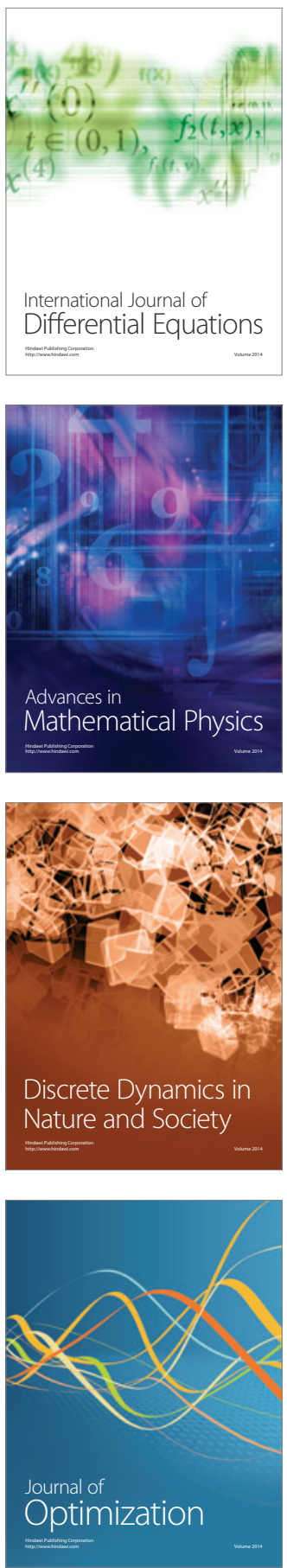